\title{
Synthesis and Spectral studies of 1,3 benzothiazole-2- thiol conjugated thiosemicarbazide as Antibacterial and Antifungal agents
}

\author{
Hiren H. Variya*, Vikram Panchal, G.R.Patel \\ Department of Chemistry, Sheth M. N. Science College, NGES campus, Patan, Gujarat-384265, India \\ E-mail: hirenvariya9@yahoo.com
}

\begin{abstract}
The newly synthesized biologically active series of 2-(benzo[d]thiazol-2-ylthio)ethanethioyl)-2(substituted)benzylidenehydrazinecarbonothioamide 6a-6f were prepared. These compounds synthesized from 1,3 benzothiazole-2-thiol, Thiosemicarbazide (TSC) and chloroaylchloride (CAC) were crucial functionalities containing the wide variety of biological activities and have a broad range of therapeutic properties. The structure of the synthesized compounds was confirmed by spectral data and evaluated for their in vitro antibacterial activities against Gram-positive and Gram-negative bacteria and antifungal
\end{abstract}

index terms-1,3 benzothiazole-2-thiol, 2-Mercaptobenzothiazole, Thiosemicarbazide, Antibacterial, Antifungal.

\section{INTRODUCTION}

Heterocyclic compounds containing $\mathrm{N}, \mathrm{O}, \mathrm{S}$, and $\mathrm{P}$ having a broad range of microbial activities against a number of bacteria and fungi. These heterocyclic compounds were used in medicinal and industrial sectors. In the medicinal applications, many heterocyclic nuclei Benzothiazole, Triazine, Benzimidazole have been considerable interest due to their important biological properties. Due to Benzothiazole possess a wide spectrum of biological activities, therefore, our interest to focus on this nucleus. Benzothiazole having potent and significant pharmacological activities such as antimicrobial [1], anticancer [2], Anthelmintic [3], Anti-diabetic [4], Anti-tuberculosis [5], antitumor [6], Antitrypanosomal [7], Anti-bacterial [8], Antiinflammatory [9], anthelmintic [10], Antifungal, antiviral, Anti-oxidant, Anti-glutamate and Antiparkinsonism, analgesic, Anti-leishmanial, Anticonvulsant [11] etc.

Thiosemicarbazide (TSC) have been considered as effective biological active molecules due to exhibit interesting pharmacological properties such as antitubercular,[12] antiviral [13], anti-malarial and antibacterial activity[14]. and anticancer [15,16], Thiosemicarbazide (TSC) and Semicarbazide (SC) are used as the key of transformation in various organic reactions and also considered as a versatile intermediate for the synthesis of important various heterocycles. With these criteria and literature data considering the importance of benzothiazole moiety in medicinal chemistry, here two series containing 2mercaptobenzothiazole and TSC were designed and synthesized for their in-vitro Antibacterial activities against Gram-positive and Gram-negative bacteria and Antifungal activities.

\section{RESULTS AND DISCUSSION}

\section{Chemistry}

The title compounds were prepared according to the synthetic strategy described in Scheme-1. The intermediate key moiety in the present work as Schiff base 3a-3f were synthesized from using various benzaldehyde and thiosemicarbazide (TSC) in the presence of acetic acid in ethanol, Further, we planned to extend our studies by using chloroaylchloride (CAC) with TEA in presence of DMF to yielded $\mathbf{4 a - 4 f}$ which treated with 2-Mercaptobenzothiazole (MBT), 1,3-Benzothiazole-2-thiol, to afford different substituent's of titled compounds 6a-6f which confirmed by spectral data.

Table 1. The substituent for derivatives of $\mathbf{6 a - 6 f}$.

\begin{tabular}{|l|l|l|l|l|}
\hline Com & \multicolumn{1}{|c|}{$\mathbf{R}$} & \multicolumn{1}{|c|}{$\begin{array}{c}\text { Molecular } \\
\text { Formula }\end{array}$} & $\begin{array}{c}\text { Yiel } \\
\mathbf{d} \\
(\%)\end{array}$ & $\begin{array}{c}\text { Melting } \\
\text { Point }^{\circ} \mathbf{C}\end{array}$ \\
\hline $\mathbf{6 a}$ & $4-\mathrm{F} \mathrm{C}_{6} \mathrm{H}_{5}$ & $\mathrm{C}_{17} \mathrm{H}_{13} \mathrm{FN}_{4} \mathrm{OS}_{3}$ & 77 & $162-165$ \\
\hline $\mathbf{6 b}$ & $4-\mathrm{Cl} \mathrm{C}_{6} \mathrm{H}_{5}$ & $\mathrm{C}_{17} \mathrm{H}_{13} \mathrm{ClN}_{4} \mathrm{OS}_{3}$ & 75 & $163-165$ \\
\hline $\mathbf{6 c}$ & $\begin{array}{l}4-\mathrm{OCH}_{3} \\
\mathrm{C}_{6} \mathrm{H}_{5}\end{array}$ & $\mathrm{C}_{18} \mathrm{H}_{16} \mathrm{~N}_{4} \mathrm{O}_{2} \mathrm{~S}_{3}$ & 72 & $165-168$ \\
\hline $\mathbf{6 d}$ & $\begin{array}{l}3-\mathrm{NO}_{2} \\
\mathrm{C}_{6} \mathrm{H}_{5}\end{array}$ & $\mathrm{C}_{17} \mathrm{H}_{13} \mathrm{~N}_{5} \mathrm{O}_{3} \mathrm{~S}_{3}$ & 70 & $162-165$ \\
\hline $\mathbf{6 e}$ & $\begin{array}{l}\mathrm{p}-\mathrm{OH} \\
\mathrm{C}_{6} \mathrm{H}_{5}\end{array}$ & $\mathrm{C}_{17} \mathrm{H}_{14} \mathrm{~N}_{4} \mathrm{O}_{2} \mathrm{~S}_{3}$ & 74 & $168-171$ \\
\hline 6f & $2,4-(\mathrm{Cl}) 2-$ & $\mathrm{C}_{17} \mathrm{H}_{12} \mathrm{Cl}_{2} \mathrm{~N}_{4} \mathrm{O}_{3}$ & 73 & $170-173$ \\
$\mathrm{C}_{6} \mathrm{H}_{5}$ & & & & \\
\hline
\end{tabular}




\section{Available online at www.ijrat.org}

\subsection{Antibacterial}

All the synthesized compounds (6a-6f) were carried out for their antibacterial activity on nutrient-agar plates by well-diffusion assay method compared to test culture. Cultures were produced in Nutrient broth. Isolates inhibit the above-mentioned organisms or not were studied and zone, of inhibition, was measured in terms of Zone diameter and with the help of that zone, the index was calculated where streptomycin was used as standard drug.

Note: Standard drug used streptomycin with 1000 $\mu \mathrm{g} / \mathrm{ml}$ concentration.

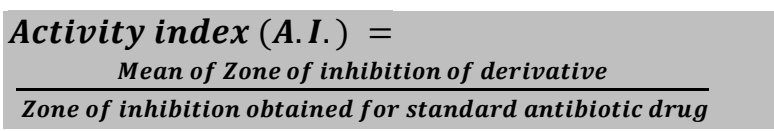

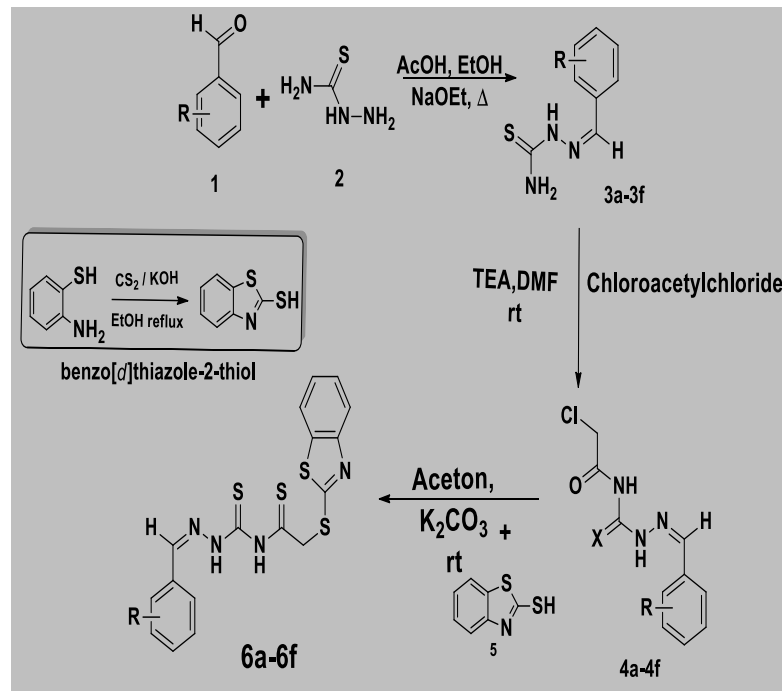

The scheme -1 Synthesis of targeted compound 6a-6f

Eq.(1) Calculate Activity index for inhibition

Table 2 Antibacterial activity of compounds $6 \mathbf{6}-\mathbf{6 f}$

\begin{tabular}{|c|c|c|c|c|c|c|c|c|}
\hline \multirow[b]{2}{*}{ Com. } & \multicolumn{2}{|c|}{$\begin{array}{c}\text { E. aerogens } \\
\text { MTCCNo. } 8558\end{array}$} & \multicolumn{2}{|c|}{$\begin{array}{c}\text { E. coli } \\
\text { MTCCNo. } 1610\end{array}$} & \multicolumn{2}{|c|}{$\begin{array}{c}\text { M. luteus } \\
\text { MTCCNo. } 11948\end{array}$} & \multicolumn{2}{|c|}{$\begin{array}{c}\text { B. cereus } \\
\text { MTCCNo. } 8558\end{array}$} \\
\hline & $\begin{array}{l}\text { Mean value } \\
\text { for Zone of } \\
\text { Inhibition } \\
\quad(\mathrm{mm})\end{array}$ & $\begin{array}{c}\text { Active } \\
\text { index } \\
\text { (A.I) }\end{array}$ & $\begin{array}{l}\text { Mean value } \\
\text { for Zone of } \\
\text { Inhibition } \\
\quad(\mathrm{mm})\end{array}$ & $\begin{array}{c}\text { Active } \\
\text { index } \\
\text { (A.I) }\end{array}$ & $\begin{array}{l}\text { Mean value } \\
\text { for Zone of } \\
\text { Inhibition } \\
(\mathrm{mm})\end{array}$ & $\begin{array}{l}\text { Active } \\
\text { index } \\
\text { (A.I) }\end{array}$ & $\begin{array}{l}\text { Mean value } \\
\text { for Zone of } \\
\text { Inhibition } \\
\text { (mm) }\end{array}$ & $\begin{array}{c}\text { Active } \\
\text { index } \\
\text { (A.I) }\end{array}$ \\
\hline Std & 25 & -- & 25 & -- & 25 & -- & 25 & -- \\
\hline $6 \mathbf{6}$ & 29 & 1.16 & 28 & 1.12 & 27 & 1.08 & 30 & 1.20 \\
\hline $\mathbf{6 b}$ & 24 & 1.041 & 25 & 1.00 & 24 & 1.041 & 22 & 0.88 \\
\hline 6c & 21 & 0.84 & 20 & 0.80 & 18 & 0.72 & 20 & 0.80 \\
\hline 6d & 26 & 1.04 & 24 & 1.041 & 26 & 1.04 & 23 & 0.92 \\
\hline $6 e$ & 21 & 0.84 & 20 & 0.80 & 22 & 0.88 & 18 & 0.72 \\
\hline 6f & 32 & 1.28 & 30 & 1.20 & 32 & 1.28 & 28 & 1.12 \\
\hline
\end{tabular}

The newly synthesized compounds 6a-6f were screened in vitro for antibacterial activity against Gram-positive Micrococcus luteus (MTCC No. 11948), Bacillus Cereus (MTCC No. 8558) and Gramnegative Enterobacter aerogens (MTCC No. 8558), Escherichia coli (MTCC No. 1610) by determining the zone of inhibition in $\mathrm{mm}$. Antibacterial showing results (the zone of inhibition), existing in Table 2. Shown that all compounds established some degree of antibacterial activity. (figure-1) The antibacterial activity of all compounds except $\mathbf{6 c}$ and $\mathbf{6 e}$ were showed less inhibition than the standard. While, compound 6a, 6d, and $6 \mathbf{f}$ have shown very close and increase activity to the standard drug. The compound 6f exhibit an excellent in all.

\subsection{Antifungal}

In this present work, all new Synthesized of series 2(benzo[d]thiazol-2-ylthio)ethanethioyl)-2-(substituted) benzylidenehydrazinecarbonothioamide 6a-6f were evaluated as antifungal activity against Aspergillus niger and Candida albicans in DMF, this activity is done by in vitro agar well diffusion method. Pepton (1g) D-glucose (4g) and agar (2g) were used to prepared Saubourauds agar media and maintained 5.7 $\mathrm{pH}$ by adding $100 \mathrm{ml}$ of distilling water and make a suspension for fungal strain. On the other hand The making suspension of corresponding species, the fungal transferred into $3 \mathrm{ml}$ salin and create a disc by adding $20 \mathrm{ml}$ of fungal media for each Petri dish after the plate was dried by using incubator at $37{ }^{\circ} \mathrm{C}$ for 1 day. A prepared control was allowed for three to four day at $37^{\circ} \mathrm{C}$ and the fungal inhibitions zone was 


\section{Available online at www.ijrat.org}

measured was the microorganism inhibited after the incubation was done and were compared with standard voriconazole shown in Table 3.

Table 3 Antifungal activity of compounds $\mathbf{6 a - 6 f}$

\begin{tabular}{|c|c|c|c|c|}
\hline \multirow{2}{*}{ Com. } & \multicolumn{2}{|c|}{ Aspergillus niger } & \multicolumn{2}{c|}{ Candida albicans } \\
\cline { 2 - 5 } & $\begin{array}{c}\text { M.V } \\
(\mathrm{mm})\end{array}$ & (A.I.) & $\begin{array}{l}\text { M.V } \\
(\mathrm{mm})\end{array}$ & (A.I.) \\
\hline 6a & 29 & 1.035 & 27 & 1.125 \\
\hline 6b & 26 & 0.929 & 20 & 0.714 \\
\hline $\mathbf{6 c}$ & 18 & 0.643 & 16 & 0.666 \\
\hline $\mathbf{6 d}$ & 26 & 0.929 & 30 & 1.250 \\
\hline $\mathbf{6 e}$ & 24 & 0.857 & 23 & 0.958 \\
\hline 6f & 30 & 1.071 & 31 & 1.291 \\
\hline Std & 28 & - & 24 & - \\
\hline
\end{tabular}

The resulted of these Antifungal activities compounds 6a, 6d and $6 \mathbf{f}$ showed excellent inhibitions as an antifungal agent.

\section{MATERIALS AND METHODS}

\subsection{Materials}

All starting materials and other reagents were purchased from commercially supplier. All the melting points were determined in open capillary and uncorrected by using a Melt-Tempt instrument, The absorbance spectra IR were recorded on Perkin-Elmer 377 spectrophotometer, ${ }^{1} \mathrm{H} \quad \mathrm{NMR}$ spectra were recorded on Bruckner at $400 \mathrm{MHz}$, In DMSO solution. The elemental analysis was analyzer for $\mathrm{C}, \mathrm{H}, \mathrm{N}, \mathrm{O}$, and $\mathrm{S}$ were estimated on PerkinElmer, series II, 2400 CHNS analyzer (CSIR Bhavnagar, INDIA) Mass spectra were recorded at Advion Expression CMS, USA

\subsection{General Procedure for targeted compounds}

(Substituted) 2-benzylidenehydrazinecarbothioamide derivatives. (3a-3f)

The aqueous Solution of thiosemicarbazide $(0.01 \mathrm{~mol})$ and benzaldehyde derivatives $(0.01 \mathrm{~mol})$ in $1: 1$ molar ratio taken in an $\mathrm{RBF}$. The reaction mixture was kept over a magnetic stirrer and stirred well at room temperature for $2 \mathrm{~h}$. the solid was formed, filtered and washed with petroleum ether. Obtained solid was dried and checked by TLC.

(Substituted) 2-benzylidene-N-(2-chloroacetyl) hydrazinecarboxamide derivatives. (4a-4f)

A mixture of compound 1(0.01 mol) with Chloroacetyl chloride $(0.015 \mathrm{~mol})$ and $4-5$ drops of TEA(triethylamine) in $25 \mathrm{ml} \mathrm{DMF}$ as the solvent in RBF. The reaction mixture was stirred for $4 \mathrm{hr}$ at Room temperature. The Completion of the reaction was Checked by TLC using toluene: Acetone (30\%). The solution poured into ice water. Obtained solid was filtered by the vacuum pump and crystalline it in Ethanol.

2-(benzo[d]thiazol-2-ylthio)ethanethioyl)-2(substituted)benzylidenehydrazinecarbonothioami de 6a-6f

Above synthesized derivatives $(0.01 \mathrm{~mol})$ was reacted with 2-mercaptobenzothiazole $(0.01 \mathrm{~mol})$ in the presence of potassium carbonate $(0.02 \mathrm{~mol})$ and acetone. The reaction was stirred at room Temperature for $5 \mathrm{hr}$. The Completion of the reaction was checked by TLC using toluene: Acetone (20\%). The product was poured into water and stirred for $1 \mathrm{hr}$. The obtained solid was collected and dried. Crystallize into ethanol.

\section{SPECTRAL DATA}

$4.12-($ benzo[d]thiazol-2-ylthio)- $\mathrm{N}-(2-(4-$ fluorobenzylidene)hydrazinecarbonothioyl)acetamide (6a) I.R (KBr) (vmax, cm-1): $3192(\mathrm{~N}-\mathrm{H}), 3088$, $2965(\mathrm{C}-\mathrm{H}), 1668(\mathrm{C}=\mathrm{O}), 1222(\mathrm{C}=\mathrm{S}), \quad 1585,1541$, 1522, 1455, $1284(\mathrm{C}=\mathrm{N}, \mathrm{C}=\mathrm{C}), 1159,1004,925,742$, $6853456 \mathrm{~cm}^{-1}$

${ }^{1}$ H NMR (400 MHz, DMSO) $\delta 11.10(\mathrm{~s}, 2 \mathrm{H}, \mathrm{NH})$, 4.32 (s, 2H, CH2), 7.05-7.38 (m, 4H, ArH), 7.5-8.6, (m, 4H, thiazole $\mathrm{ArH}), 6.6(\mathrm{~s}, 1 \mathrm{H} \mathrm{HC}=\mathrm{N})$

${ }^{13}$ C-NMR $\delta 38.5$ (CH2), 147.8 (N-CH-Ar), 172.2 $(\mathrm{C}=\mathrm{O}), 181.4(\mathrm{C}=\mathrm{S}), 123-154.8$ (12 aromatic carbons), 167.7 (S-C-N benz thio)

MS (m/z) $389.05\left(\mathrm{M}^{+}\right)$

Anal. Calcd. For: $\mathrm{C}_{17} \mathrm{H}_{13} \mathrm{FN}_{4} \mathrm{O}_{2} \mathrm{~S}_{2} \mathrm{C}$, 52.56; H, 3.37; N, 14.42; O, 8.24; S, 16.51\% Found: C 53.7, H 3.32, N 14.16, O 8.22 S $16.58 \%$.

4.2 2-(benzo[d]thiazol-2-ylthio)-N-(2-(4chlorobenzylidene)hydrazinecarbonothioyl)acetamid $\boldsymbol{e}(\mathbf{6 b})$

I.R (KBr) (vmax, cm-1): $3196(\mathrm{~N}-\mathrm{H}), 3086,2966$ (C$\mathrm{H}), 1665(\mathrm{C}=\mathrm{O}), 1212(\mathrm{C}=\mathrm{S}), 1584,1542,1512$, 1448,1294 (C=N, C=C), 1159, 1004, 925, 742, 685 $3456 \mathrm{~cm}^{-1}$

${ }^{1} \mathbf{H}$ NMR (400 MHz, DMSO) $\delta 11.02$ (s, 2H, NH), 4.12 (s, 2H, CH2), 7.05-7.42 (m, 4H, ArH), 7.5-8.55, (m, 4H, thiazole ArH),6.6 (s, 1H HC=N)

${ }^{13}$ C-NMR $\delta 39.5$ (CH2), 145.8 (N-CH-Ar), 170.2 $(\mathrm{C}=\mathrm{O}), 180.4(\mathrm{C}=\mathrm{S}), 123-154.8$ (12 aromatic carbons), 167.7 (S-C-N benz thio),

MS (m/z) $419.06\left(\mathrm{M}^{+}\right)$

Anal. Calcd. For: $\mathrm{C}_{17} \mathrm{H}_{13} \mathrm{ClN}_{4} \mathrm{OS}_{3} \mathrm{C}$, 48.50; H, 3.11; N, 13.31; O, 3.80; S, 22.85\% Found: C 48.7, H 3.12, $\mathrm{N} 13.36, \mathrm{O} 3.88 \mathrm{~S} 22.88 \%$.

4.3

2-(benzo[d]thiazol-2-ylthio)- $\mathrm{N}-(2-$ (4methoxybenzylidene)hydrazinecarbonothioyl)aceta mide (6c) 


\section{Available online at www.ijrat.org}

I.R (KBr) (vmax, cm-1): 3192 (N-H), 3082, 2955 (C$\mathrm{H}), 1662(\mathrm{C}=\mathrm{O}), 1222(\mathrm{C}=\mathrm{S})$, 1570, 1534, 1522, 1458,1296 $(\mathrm{C}=\mathrm{N}, \mathrm{C}=\mathrm{C}), 1154,1006,924,740,684$ $3454 \mathrm{~cm}^{-1}$

${ }^{1}$ H NMR (400 MHz, DMSO) $\delta 3.71\left(\mathrm{~s}, 3 \mathrm{H} \mathrm{Ar}-\mathrm{OCH}_{3}\right)$ 11.03 (s, 2H, NH), 4.16 (s, 2H, CH2), 7.05-7.45 (m, $4 \mathrm{H}, \mathrm{ArH}), 7.5-8.55,(\mathrm{~m}, 4 \mathrm{H}$, thiazole $\mathrm{ArH}), 6.6(\mathrm{~s}, 1 \mathrm{H}$ $\mathrm{HC}=\mathrm{N}$ )

${ }^{13}$ C-NMR $\delta 39.5$ (CH2), 145.8 (N-CH-Ar), 171.2 $(\mathrm{C}=\mathrm{O}), 181.4(\mathrm{C}=\mathrm{S}), 123-154.8$ (12 aromatic carbons), 166.7 (S-C-N benz thio), $55.4\left(\mathrm{OCH}_{3}\right)$

MS (m/z) $416.05\left(\mathrm{M}^{+}\right)$

Anal. Calcd. For: $\mathrm{C}_{18} \mathrm{H}_{16} \mathrm{~N}_{4} \mathrm{O}_{2} \mathrm{~S}_{3}$, C, 53.98; H, 4.03; N, 13.99; O, 3.99; S, 24.02\% Found: C 53.7, H 4.09, N 13.96,O 3.98 S $24.08 \%$.

\subsection{2-(benzo[d]thiazol-2-ylthio)- N-(2-(4- nitrobenzylidene )hydrazinecarbonothioyl)acetamide (6d)}

I.R (KBr) (vmax, cm-1): $3192(\mathrm{~N}-\mathrm{H}), 3080,2956$ (C$\mathrm{H}), 1662(\mathrm{C}=\mathrm{O}), 1224(\mathrm{C}=\mathrm{S}), 1581,1539,1522$, 1448,1294 $(\mathrm{C}=\mathrm{N}, \mathrm{C}=\mathrm{C}), 1149,1004,921,742,684$ $3455 \mathrm{~cm}^{-1}$

${ }^{1}$ H NMR (400 MHz, DMSO) $\delta 11.11(\mathrm{~s}, 2 \mathrm{H}, \mathrm{NH})$, 4.15 (s, 2H, CH2), 7.05-7.48 (m, 4H, ArH), 7.5-8.5, $(\mathrm{m}, 4 \mathrm{H}$, thiazole $\mathrm{ArH}), 6.6(\mathrm{~s}, 1 \mathrm{H} \mathrm{HC}=\mathrm{N})$

${ }^{13}$ C-NMR $\delta 39.5$ (CH2), 145.8 (N-CH-Ar), 171.2 $(\mathrm{C}=\mathrm{O}), 181.5(\mathrm{C}=\mathrm{S}), 123-154.8$ (12 aromatic carbons), 166.7 (S-C-N benz thio)

MS (m/z) $431.12\left(\mathrm{M}^{+}\right)$

Anal. Calcd. For: $\mathrm{C}_{17} \mathrm{H}_{13} \mathrm{~N}_{5} \mathrm{O}_{3} \mathrm{~S}_{3} \mathrm{C}$, 47.32; H, 3.04; N, 16.23; O, 11.12; S, 22.29\% Found: C 47.2, H 3.12, N $16.26,011.18$ S $22.28 \%$.

4.5 2-(benzo[d]thiazol-2-ylthio)-N-(2-(4hydroxybenzylidene)hydrazinecarbonothioyl)acetami de (6e)

I.R (KBr) (vmax, cm-1): 3198 (N-H), 3088, 2965 (C$\mathrm{H}), 1668(\mathrm{C}=\mathrm{O}), 1221(\mathrm{C}=\mathrm{S}), 1585,1541,1522$, 1455,1284 $(\mathrm{C}=\mathrm{N}, \mathrm{C}=\mathrm{C}), 1159,1004,925,742,685$ $3456 \mathrm{~cm}^{-1}$;

${ }^{1}$ H NMR (400 MHz, DMSO) $\delta \quad 11.02$ (s, 2H, NH), 11.59 (s, 1H, OH), 4.32 (s, 2H, CH2), 7.05-7.38 (m, $4 \mathrm{H}, \mathrm{ArH}), 7.5-8.6,(\mathrm{~m}, 4 \mathrm{H}$, thiazole $\mathrm{ArH}), 6.6(\mathrm{~s}, 1 \mathrm{H}$ $\mathrm{HC}=\mathrm{N}$ )

${ }^{13}$ C-NMR $\delta 38.5$ (CH2), 147.8 (N-CH-Ar), 172.2 $(\mathrm{C}=\mathrm{O}), 180.5(\mathrm{C}=\mathrm{S}), 123-154.8$ (12 aromatic carbons), 167.7 (S-C-N benz thio),

MS (m/z) $402.05\left(\mathrm{M}^{+}\right)$

Anal. Calcd. For: $\mathrm{C}_{17} \mathrm{H}_{14} \mathrm{~N}_{4} \mathrm{O}_{2} \mathrm{~S}_{3} \mathrm{C}, 50.73 ; \mathrm{H}, 3.51$; N, 13.92; O, 7.95; S, 23.90\% Found: C 50.7, H 3.62, N 14.01, O 7.92 S $23.98 \%$

4.6 2-(benzo[d]thiazol-2-ylthio)- $\mathrm{N}-(2-(2,4-$ dichlorobenzylidene)hydrazinecarbonothioyl)acetami de $(6 e)$

I.R (KBr) (vmax, cm-1): $3192(\mathrm{~N}-\mathrm{H}), 3088,2965$ (C$\mathrm{H}), 1668(\mathrm{C}=\mathrm{O}), 1210(\mathrm{C}=\mathrm{S}), 1585,1541,1522$,
1455,1284 $(\mathrm{C}=\mathrm{N}, \mathrm{C}=\mathrm{C}), 1159,1004,925,742,685$ $3456 \mathrm{~cm}^{-1}$;

${ }^{1}$ H NMR (400 MHz, DMSO) $\delta 11.08$ (s, 2H, NH), 4.32 (s, 2H, CH2), 7.05-7.28 (m, 3H, ArH), 7.5-8.6, (m, 4H, thiazole $\mathrm{ArH}), 6.6(\mathrm{~s}, 1 \mathrm{H} \mathrm{HC}=\mathrm{N})$

${ }^{13}$ C-NMR $\delta \quad 38.7$ (CH2), 147.8 (N-CH-Ar), 172.4 $(\mathrm{C}=\mathrm{O}), 180.1(\mathrm{C}=\mathrm{S}), 123-155.8$ (12 aromatic carbons), 167.7 (S-C-N benz thio),

MS (m/z) $453.15\left(\mathrm{M}^{+}\right)$

Anal. Calcd. For: $\mathrm{C}_{17} \mathrm{H}_{12} \mathrm{Cl}_{2} \mathrm{~N}_{4} \mathrm{OS}_{3} \mathrm{C}$, 44.84; H, 2.66 $\mathrm{N}, 12.30 ; \mathrm{O}, 3.51 ; \mathrm{S}, 21.12$, \% Found: $\mathrm{C} 44.81, \mathrm{H}$ $2.62, \mathrm{~N} 12.36, \mathrm{O} 3.52 \mathrm{~S} 21.18 \%$

\section{CONCLUSION}

In this study, we describe the methodology for 1,3benzthiazole-2-thiol and Thiosemicarbazide (TSC) containing new derivatives 6a-6f. The pharmacological study for these two series evaluated on the in vitro antibacterial activities against grampositive and gram-negative bacteria. All compounds shown good activity with using streptomycin as standard drug except 6a, 6d, and $6 \mathbf{f}$ showed more than inhibition the standard against all bacterial strains and also being good inhibitors against fungal strains showed very close activity to standard drug.

\section{Acknowledgements}

The author is thankful and expresses sincere gratitude to The Sheth M. N. Science College, H.N.G.U., Patan. The author is also thankful Saurashtra University, Rajkot and CMCRI, Bhavnagar for providing analytical data of the compounds. 


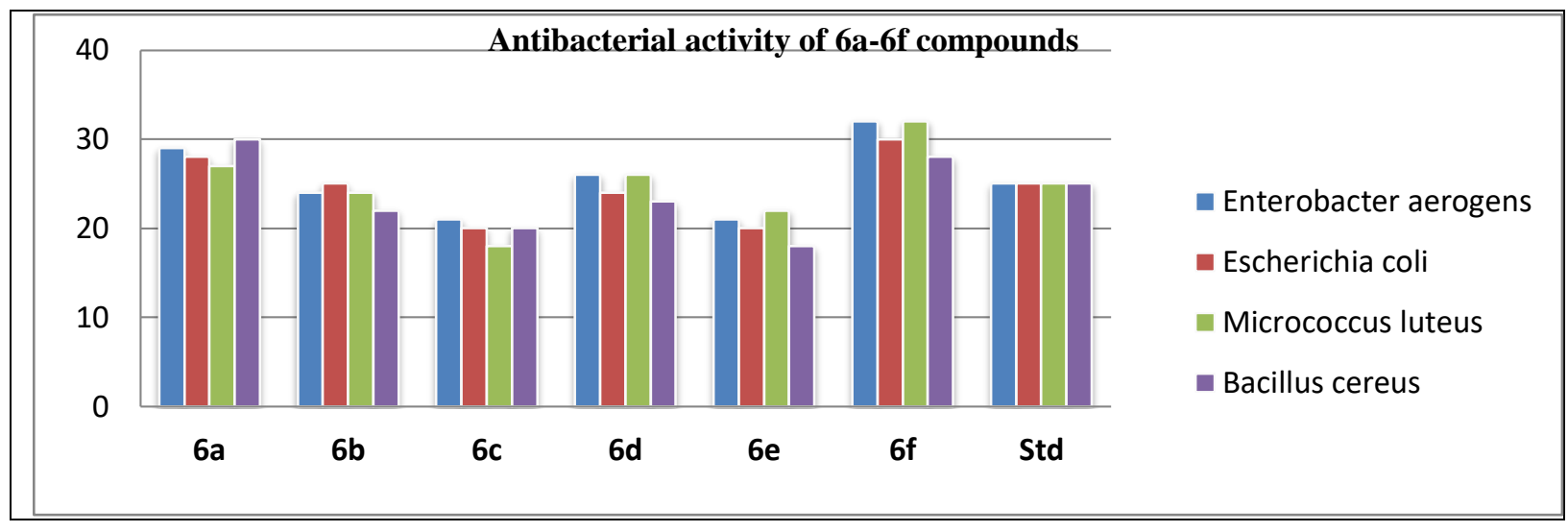

Figure1: Line scatter graph about Antibacterial activity of Compound 3a-3r for Gram-positive Micrococcus luteus (MTCC No. 11948), Bacillus Cereus (MTCC No. 8558) and Gram-negative Enterobacter aerogens (MTCC No. 8558), Escherichia coli (MTCC No. 1610)

\section{REFERENCES}

[1] S. Gupta, N. Ajmera. (2009): Solvent-free synthesis of tetrazoles, Indian J. Chem., Sect. B: Org. Chem. Incl. Med. Chem., 48 853-863.

[2] SHL. Kok, R. Gambari, CH. Chui, MCW. Yuen, E. Lin, RSM. Wong, et al. (2008): Synthesis and anticancer activity of benzothiazole containing phthalimide on human carcinoma cell lines, Bioorg. Med. Chem., 16 3626-3631.

[3] G. Sreenivasa, E. Jayachandran, B. Shivakumar, K. Jayaraj, M. Kumar Vijay. (2009): Synthesis of bioactive molecule fluoro benzothiazole comprising potent heterocyclic moieties for anthelmintic activity, Arch Pharm Sci \& Res 1150-157.

[4] Pattan SR, Suresh C, Pujar VD, Reddy VVK, Rasal VP, Kotti BC. (2005): Indian J Chem 4B 2404.

[5] J. Das, RV. Moquin, J. Lin, C. Liu, AM. Doweyko, HF. DeFex, et al. (2003): Discovery of 2-amino-heteroaryl-benzothiazole-6-anilides as potent p56 lack inhibitors, Bioorg. Med. Chem. Lett., 13 2587-2590.

[6] T. Bradshaw, A. Westwell. (2004): The development of the antitumour benzothiazole prodrug, Phortress, as a clinical candidate, Curr. Med. Chem., 11 1009-1021.

[7] J. Neres, ML. Brewer, L Ratier, H. Botti, A. Buschiazzo, PN. Edwards, et al. (2009): Discovery of novel inhibitors of Trypanosoma cruzitrans-sialidase from in silico screening, Bioorg. Med. Chem. Lett., 19 589-596.

[8] Russo F, Santagati M. (1976) Farmaco Ed Sci 31 41.

[9] Sawhney SN, Bansal OP. (1977): Indian J Chem 15B 121.
[10] V.P.Devmurari, T.J.Ghodasara. (2010): Synthesis and antibacterial activity of some substituted 2phenyl benzothiazole, Arch. Appl. Sci. Res. 2 198-203.

[11] MN. Bhoi, MA. Borad, HD. Patel. (2014) Synthetic Strategies for Fused Benzothiazoles: Past, Present, and Future. Synth Com., 44 24272457.

[12] Sriram D, Yogeeswari P, Thirumurugan R and Pavana RK. (2006): Discovery of new antitubercular oxazolyl thiosemicarbazones. J. Med. Chem. 49 3448-3450.

[13] Garcia CC, Brousse BN, Carlucci MJ, Moglioni AG, Martins AM, Moltrasio GY, Daccorso NB and Damonte EB. (2003): Inhibitory effect of thiosemicarbazone derivatives on Junin virus replication in-vitro. Antivir. Chem. Chemother. 14 99-105.

[14] Klayman DL, Scovill JP, Bruce J and Bartosevich JF. (1984): 2-Acethylpyridine thiosemicarbazones derivatives of acethylisoquinoline as potential antimalarial agents. J. Med. Chem. 27 84-87.

[15] Yu Y, Kalinowski DS, Kovacevic Z, Siafakas AR, Jansson PJ, Stefani C, Lovejoy DB, Sharpe PC, Bernhardt PV and Richardson DR. (2009): Thiosemicarbazones from the old to new: Iron chelators that are more than just ribonucleotide reductase inhibitors. J. Med. Chem. 52 52715294.

[16] Jalilian AR, Yousefnia H, Shafaii K, Novinrouz Aand Rajamand AA. (2012): Preparation and biodistribution studies of a radiogalliumacetylacetonatebis (thiosemicarbazone) complex in tumour-bearing rodents. Iran. J. Pharm. Res. 11 523-531. 